\title{
Telomere clustering drives ALT
}

Jaewon Min and Jerry W. Shay

Advanced human tumor cells universally need to acquire a telomere maintenance mechanism (TMM). While almost all carcinomas acquire a TMM by upregulating telomerase, there are some tumors that can divide indefinitely without activating telomerase. This is especially true in cancers of mesenchymal origin (such as sarcomas, endocrine tumors, glioblastoma, and some early childhood cancers). This telomeraseindependent TMM has been termed Alternative Lengthening of Telomeres (ALT) [1]. ALT is a recombination-based telomere maintenance mechanism, however the underlying mechanisms by which the ALT pathway is initiated/mediated are still poorly understood. One of the hallmarks of ALT positive cancer specimens is the excessively clustered telomeres, shown as large bright telomere foci [2]. These telomere signals are clustered in promyelocytic leukemia (PML) bodies,

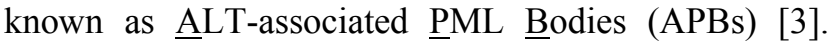
APBs contain telomeric DNA as well as many proteins involved in DNA replication, repair, and recombination processes. PML bodies are one of the nuclear membrane-less organelles which form by liquid-liquid phase separation organized by multivalent protein

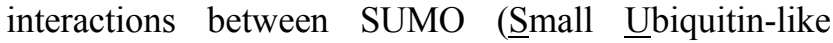
MOdifier) and SIM (UMO-Interacting Motif) [4]. The underlying mechanisms by which telomere clustering in PML bodies are induced and how this is linked to the ALT pathway are not well understood.

We recently demonstrated a newly developed biophysical system that reconstitutes PML bodies from minimal components and generates telomere clustering, thus mimicking APBs in vivo [5]. By using polySUMO/ polySIM condensates targeting telomeres, we demonstrated that the ALT mechanism in humans is triggered by excessive and persistent clustering of telomeres in PML bodies. Our observations help explain why ALT cancers display large bright telomere foci. Specifically, we demonstrated that these telomere foci are involved in telomere elongation processes. We further showed that the molecular mechanism of ALT is mediated by two stepwise functions of the BLM an RAD52 genes. ALT-like phenotypes are rapidly induced by introducing the APB-like condensates together with BLM overexpression in the presence of endogenous RAD52. We further determined that the underlying mechanism was associated with the helicase activity of BLM protein that is involved in 5' to 3' resection processes.
In addition, the multimerization and DNA binding activity of the RAD52 protein, which has annealing activity, also participates in the ALT pathway. We demonstrated that the helicase activity of BLM is required for the initiation of telomere clustering and telomere synthesis through the generation of singlestranded telomeric DNAs via long-range resection processes. Consistent with our observations, it has been shown that the helicase activity of BLM is essential for the ALT activity in other organisms, i.e. S. cerevisiae and $U$. maydis [6]. We further demonstrated that RAD52 participates in ALT processes through its highly conserved N-Terminal Domain (NTD). The RAD52 NTD has the annealing activity between singlestranded DNA and potential templates, such as single stranded DNA, double stranded DNA, or RNA. Collectively, we proposed that RAD52 is involved in annealing the resected single-stranded telomeres to potential templates, i.e. chromosomal telomeres, extrachromosomal telomeric repeats, and telomeric noncoding RNA (TERRA), leading to telomere elongation through non-canonical recombination processes, such as break-induced replication, rolling circle amplification, and RNA-templated DNA repair (Figure 1).

Human ALT cancer cells exhibit severe DNA replication stresses at telomeres, which leads to SMC5/6-mediated SUMOylation at telomeres and telomere clustering in PML bodies (e.g. formation of APBs) [7]. Clustered telomeres in APBs are processed by long-rang resection reactions though BLM-DNA2 (helicase-endonuclease) and possibly other exonucleases (i.e. MRE11) [6,7] leading to the accumulation of single-stranded telomeric DNAs. Since most ALT cells do not have an intact G2/M checkpoint, ALT cells can bypass the checkpoint and undergo cell cycle progression with these exposed single-stranded telomeres [8]. Ultimately, these excessive amounts of single stranded telomeres clustered in APBs are repaired in late G2 or early M-phase by non-canonical recombination mediated by RAD52, whereas canonical recombination processes (i.e. homologous recombination) are suppressed by highly-enriched CDK activity in late G2/early M.

In conclusion, we generated artificially engineered APB-like condensates in vivo to study the ALT pathway [5]. Further refining our model system to more closely reflect spontaneous in vivo events (i.e. 
continuous cell proliferation and inducing telomere clustering in endogenous PML bodies) will provide further insights into the ALT pathway. Since APBs are the major site for telomeric synthesis during late G2/early M in ALT cells, a better understanding of the regulation of APB assembly/disassembly processes are likely to be crucial for the development of novel therapeutic strategies for ALT cancers in the future.
7. Min J, et al. Mol Cell Biol. 2017; 37. https://doi.org/10.1128/MCB.00226-17 PMID: 28760773

8. Min J, et al. Nucleic Acids Res. 2017; 45:2615-28. https://doi.org/10.1093/nar/gkw1295 PMID:28082393

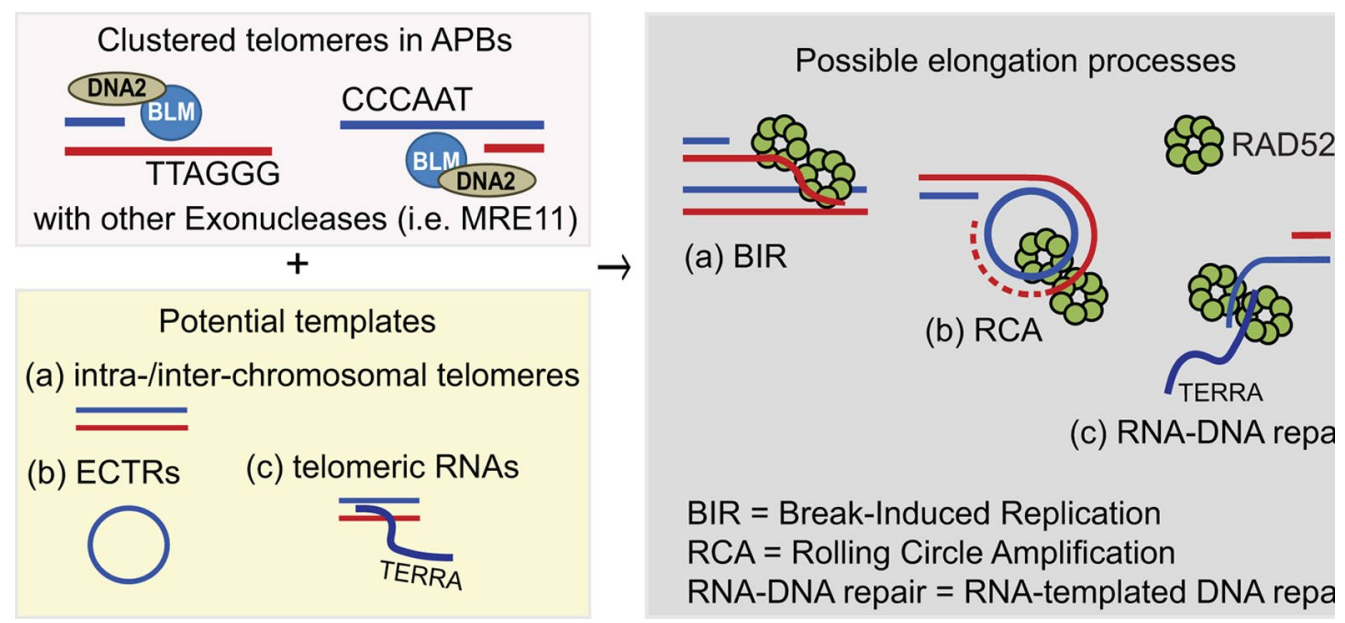

Figure 1. Schematic models for human ALT pathway.

\section{REFERENCES}

1. Bryan TM, et al. Nat Med. 1997; 3:1271-74. https://doi.org/10.1038/nm1197-1271 PMID: 9359704

2. Heaphy CM, et al. Science. 2011; 333:425. https://doi.org/10.1126/science.1207313 PMID:21719641

3. Yeager TR, Neumann AA, Englezou A, Huschtscha LI, Noble JR, Reddel RR. Telomerase-negative immortalized human cells contain a novel type of promyelocytic leukemia (PML) body. Cancer Res. 1999; 59:4175-79. PMID:10485449

4. Banani SF, et al. Cell. 2016; 166:651-63. https://doi.org/10.1016/j.cell.2016.06.010 PMID:27374333

5. Min J, et al. Genes Dev. 2019; 33:814-27. https://doi.org/10.1101/gad.324905.119 PMID:31171703

6. Yu EY, et al. PLoS Genet. 2015; 11:e1005570. https://doi.org/10.1371/journal.pgen.1005570 PMID:26492073
Jaewon Min: Department of Cell Biology, University of Texas Southwestern Medical Center, Dallas, TX 75390, USA

Correspondence: Jaewon Min

Email: jaewon.min@utsouthwestern.edu

Keywords: telomeres, telomerase, BLM, RAD52, MiDAS, phase-separation, biomolecular condensates, $P M L$ bodies

Acknowledgements: We thank Neal F. Lue (Weill Cornell Medicine) for discussion

Funding: This work was supported by AG01228 from the National Institute on Aging (J.W.S). We also acknowledge the Harold Simmons $\mathrm{NCl}$ Designated Comprehensive Cancer Center Support Grant (CA142543), and the Southland Financial Corporation Distinguished Chair in Geriatric Research (J.W.S.)

Copyright: Min and Shay. This is an open-access article distributed under the terms of the Creative Commons Attribution License (CC BY 3.0), which permits unrestricted use, distribution, and reproduction in any medium, provided the original author and source are credited

Received: August 29, 2019

Published: October 13, 2019 